\title{
Ventilator-associated pneumonia and bloodstream infections in intensive care unit cancer patients: a retrospective 12-year study on 3388 prospectively monitored patients
}

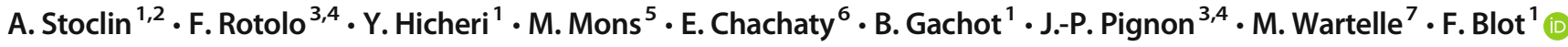

Received: 19 October 2018 / Accepted: 3 April 2019 / Published online: 17 April 2019

(C) Springer-Verlag GmbH Germany, part of Springer Nature 2019

\begin{abstract}
Purpose Some publications suggest high rates of healthcare-associated infections (HAIs) and of nosocomial pneumonia portending a poor prognosis in ICU cancer patients. A better understanding of the epidemiology of HAIs in these patients is needed.

Methods A retrospective analysis of all the patients hospitalized for $\geq 48 \mathrm{~h}$ during a 12 -year period in the 12-bed ICU of the Gustave Roussy hospital, monitored prospectively for ventilator-associated pneumonia (VAP) and bloodstream infection (BSI) and for use of medical devices.

Results During 3388 first stays in the ICU, 198 cases of VAP and 103 primary, 213 secondary, and 77 catheter-related BSIs were recorded. The VAP rate was $24.5 / 1000$ ventilator days (95\% confidence interval [CI] 21.2-28.0); the catheter-related BSI rate was $2.3 / 1000$ catheter days $(95 \%$ CI 1.8-2.8). The cumulative incidence during the first 25 days of exposure was $58.8 \%(95 \% \mathrm{CI}$ 49.1-66.6\%) for VAP, $8.9 \%$ (95\% CI, 6.2-11.5\%) for primary, $15.1 \%$ (95\% CI 11.6-18.5\%) for secondary and $5.0 \%$ (95\% CI 3.2-6.8\%) for catheter-related BSIs. VAP or BSIs were not associated with a higher risk of ICU mortality.

Conclusions This is the first study to report HAI rates in a large cohort of critically ill cancer patients. Although both the incidence of VAP and the rate of BSI are higher than in general ICU populations, this does not impact patient outcomes. The occurrence of device-associated infections is essentially due to severe medical conditions in patients and to the characteristics of malignancy.
\end{abstract}

Keywords Healthcare-associated infections - Intensive care unit - Risk factors - Catheter-associated infections · Ventilator-associated pneumonia

\section{Introduction}

Intensive care unit (ICU) patients develop life-threatening healthcare-associated infections (HAIs) more frequently than other patients due to their acute illness and invasive procedures. Infection surveillance networks provide comparative HAI data that can be adjusted, at least partially, for intrinsic and extrinsic risk factors in patients [1]. The HAI rates differ
Electronic supplementary material The online version of this article (https://doi.org/10.1007/s00520-019-04800-6) contains supplementary material, which is available to authorized users.

A. Stoclin

annabelle.stoclin@gustaveroussy.fr

1 Service de Médecine Intensive Réanimation, Gustave Roussy, 114, rue Edouard Vaillant, Villejuif 94805, France

2 Service de Réanimation Médico-Chirurgicale, Gustave Roussy, 114, rue Edouard Vaillant, 94805 Villejuif, France

3 Service de Biostatistique et d'Epidémiologie, Gustave Roussy, 114, rue Edouard Vaillant, Villejuif 94805, France
$4 \quad$ INSERM U1018, CESP, Université Paris-Sud, Université Paris-Saclay, Villejuif, France

5 Service d'Information Médicale, Gustave Roussy, 114, rue Edouard Vaillant, Villejuif 94805, France

6 Service de Microbiologie Médicale, Gustave Roussy, 114, rue Edouard Vaillant, Villejuif 94805, France

7 Direction du Système d'Information, Gustave Roussy, 114 Rue Edouard Vaillant, Villejuif 94805, France 
according to the ICU type and patient mix [1]. Among the HAIs, ventilator-associated pneumonia (VAP), clinical sepsis and bloodstream infections (BSIs) are associated with a poorer prognosis [2].

A growing number of cancer patients are admitted to ICUs and regular improvement of their prognosis has been observed [3]. The rare publications available show high HAI rates [4] and the poor prognosis of nosocomial pneumonia in critically ill cancer patients [5]. There is a need for a better understanding of the epidemiology, risk factors, and outcomes concerning HAIs in this population.

\section{Patients and methods}

Our main objective was to report the incidence of VAPs and BSIs in critically ill cancer patients based on a 12-year prospective cohort in our oncology ICU. Our secondary objectives were to describe pathogen distribution and assess the risk factors for HAIs and their potential influence on ICU mortality.

The Gustave Roussy Cancer Centre is a tertiary care hospital treating exclusively patients with solid or hematological malignancies. The average annual admission volume in the 12-bed medical surgical ICU is 400 to 450 patients. A dedicated infection control team is in place since 1999.

We collected data from the hospital activity and associated expenditure database (Programme de Médicalisation des Systèmes d'Information, PMSI) and from the ICU case report forms for stays $\geq 48 \mathrm{~h}$.

The PMSI national database contains information on admission categories, patient demographics, disease characteristics, Eastern Cooperative Oncology Group performance status (ECOG-PS [6]), and Simplified Acute Physiology Score (SAPS II [7]).

The ICU registry is based on a questionnaire, filled out by the same two physicians since 1999. The case report forms include information on the following: invasive devices (mechanical ventilation $[\mathrm{MV}]$ and central venous catheters [CVCs]), HAIs (VAP, primary BSIs, catheter-related BSIs, and secondary BSIs), neutropenia (white blood cell [WBC] count $<1000 / \mathrm{mm}^{3}$ or acute leukemia) before admission (duration and nadir), and outcomes at discharge from ICU (infections [date of diagnosis, pathogen] and death. MV by intubation or tracheotomy and CVCs (including totally implanted ports and hemodialysis lines) were studied. As the majority of long-term CVCs were used throughout the ICU stay, we did not differentiate between long-term and temporary central lines. All patients were prospectively monitored for infections from admission to $48 \mathrm{~h}$ after discharge. All the ICU stays between January 1, 2000, and December 31, 2011, were considered. During this period, the main change in routine practice was the use of a sedation scale for mechanically ventilated patients since 2004 . The data are strictly confidential and available only to authorized clinicians and staff. (Medical procedures are described in the supplementary material)

Diagnostic techniques and infection criteria remained unchanged during the study period. All cases of pneumonia and BSIs were audited by two authors (AS, FB) in 2013-2014, using the microbiology data and medical records. All clinically suspected VAP were confirmed using a quantitative culture of distal respiratory tract secretions, "blindly," or via a fiberoptic bronchoscope (or sometimes a semi-quantitative culture of sputum after extubation). For details, see web supplements. Bacteremia or fungemia were defined as at least one positive blood culture (except for skin commensals). BSIs were classified as primary, secondary, or catheter-related BSI (CR-BSI) (see supplementary material).

\section{Statistical analysis}

We retrospectively described the use of invasive devices in terms of the number of devices, median placement time, and inter-quartile ranges (IQRs). Device usage rates (ratio of the duration of device use to the duration of the stays) were calculated separately for the first (per patient) ICU stays and for the remaining stays. We computed the rates of HAIs as 1000 times the ratio of the total number of infections to the total number of ICU days. HAI rates were calculated as $(1000 \times)$ the ratio of the total number of infections (VAP and CR-BSIs) to the total duration of the device.

For patients with several stays exceeding $48 \mathrm{~h}$, only the first stay was included in the prognostic analyses. Analogously, only the first HAI was considered. To verify whether the infection risk was constant over time, we compared the exponential estimation of the cumulative incidence to the $95 \%$ confidence bands of the Kaplan-Meier estimate.

We identified factors associated with infection using logistic models adjusted for the exposure time. We computed univariate models, then multivariate models via stepwise selection based on likelihood ratio tests $\left(\alpha_{\text {in }}=0.2, \alpha_{\text {out }}=0.05\right)$. The methods used for sensitivity analyses and factors associated with mortality are shown in the supplementary material. All analyses were performed using SAS 9.3 and R 3.1.

\section{Results}

The number of ICU stays was 4554, of which 554 lasted $<$ $48 \mathrm{~h}$ (flowchart: Figure S1). The number of first stays $\geq 48 \mathrm{~h}$ was 200 to 300 per year until 2008 , then it increased. The median length of the ICU stay was constantly about 67 days (Figure S2).

Table 1 describes patient characteristics. Among the 4000 stays $\geq 48$ h, 3388 were first stays (median duration 6 days). Most of the patients $(1938 ; 57 \%)$ were admitted for medical 
reasons; 2640 (79\%) had solid tumors, and 1461 (43\%) had metastatic disease. At ICU admission, 320 patients $(9 \%)$ had experienced leukopenia, for more than 7 days in $108(3 \%)$ cases. The ICU mortality rate was constantly $14 \%$.

\section{Invasive devices}

Among the first stays $(N=3388)$, 930 patients $(27 \%)$ experienced one or more episodes of MV (median duration 4 days) and 2806 patients $(83 \%)$ underwent at least one CVC placement (median dwell time 7 days; le S1). The total CVC dwell time $(33,498$ days) exceeded the total duration of stays (31,670 days) because most patients had several catheters, including CVCs (preexisting and implanted in the ICU). The ICU device usage rate was $25.5 \%$ for MV and $105.8 \%$ for CVCs. Figure 1 shows the yearly number of stays $\geq 48 \mathrm{~h}$ with devices and median device duration.

\section{Healthcare-associated infections}

During the first stays (Table 2), we recorded 198 cases of VAP ( $N=153$ patients) and 393 BSIs $(N=296)$ : 103 were primary $(N=96), 77$ CR-BSIs $(N=73)$, and 213 secondary $(N=171)$.

The VAP rate was $24.5 / 1000 \mathrm{MV}$ days $(95 \%$ confidence interval [CI] 21.2-28). This rate varied remarkably over the years (Figure S3, left). CVCs were responsible for $19.5 \%$ of all BSIs (77/393). The CR-BSI rate was 2.3/1000 CVC days (95\% CI 1.8-2.8), with small variations over time (Figure S3, right; Fig. 2).

The most common secondary BSIs were of abdominal origin $(58.7 \%, 125 / 213)$. Thirty-four BSIs were due to VAP and 23 to urinary tract infections.

Figure 3 shows the number of stays with at least one HAI of each type. Among the 379 patients with infections, $75 \%$ (286) experienced only one type of infection, 73 two types, 19 three, and 1 all four types.

\section{Microbiologic findings}

The most common pathogens (Table S2) were Gram-negative aerobes bacilli (373 isolates, 49.8\%) and Gram-positive cocci (271 isolates, 36.2\%). Candida species and other fungi accounted for 34 isolates $(4.5 \%)$. Polymicrobial infections were recorded in 37/153 (24.2\%) VAPs (first episodes) and 35/340 (10.3\%) BSIs: 6/96 (6.3\%) primary BSIs, 20/171 (11.7\%) secondary BSIs, and 9/73 (12.3\%) CR-BSIs. Almost $5.5 \%$ of bloodstream isolates $(N=24)$ were Candida species, $29 \%(N=7)$ of which were Candida albicans.

\section{Risk factors for infections}

Figure 2 shows the cumulative incidence rates of VAP and primary and secondary BSIs and CR-BSIs. The results of
Table 1 Patient characteristics

\begin{tabular}{|c|c|c|}
\hline & 1st stays & 2 nd + stays \\
\hline \multicolumn{3}{|l|}{ ICU stays } \\
\hline Number of stays & 3388 & 612 \\
\hline Total duration (days) & 31,670 & 5798 \\
\hline Median duration (q25-q75) & $6(3-10)$ & $6(3-10)$ \\
\hline \multicolumn{3}{|l|}{ Sex } \\
\hline Male & $1844(54 \%)$ & $364(59 \%)$ \\
\hline Female & $1544(46 \%)$ & $248(41 \%)$ \\
\hline \multicolumn{3}{|l|}{ Age (years) } \\
\hline Median (q25-q75) & $56(46-65)$ & $55(46-64)$ \\
\hline \multicolumn{3}{|l|}{ SAPS II score } \\
\hline Median (q25-q75) & $31(22-43)$ & $34(25-43)$ \\
\hline \multicolumn{3}{|l|}{ Admission category } \\
\hline Medical & $1938(57 \%)$ & $454(74 \%)$ \\
\hline Scheduled surgery & $1083(32 \%)$ & $54(9 \%)$ \\
\hline Unscheduled surgery & $367(11 \%)$ & $104(17 \%)$ \\
\hline \multicolumn{3}{|l|}{ Type of neoplasia } \\
\hline Benign tumor & $110(3 \%)$ & $14(2 \%)$ \\
\hline Hematological malignancy & $606(18 \%)$ & $114(19 \%)$ \\
\hline Solid tumor & $2640(79 \%)$ & $478(79 \%)$ \\
\hline Missing data, $N$ & 32 & 6 \\
\hline \multicolumn{3}{|l|}{ Presence of metastases } \\
\hline No & $1927(57 \%)$ & $336(55 \%)$ \\
\hline Yes & $1461(43 \%)$ & $276(45 \%)$ \\
\hline \multicolumn{3}{|l|}{ WHO score } \\
\hline $0 / 1 / 2$ & $1842(56 \%)$ & $249(41 \%)$ \\
\hline $3 / 4$ & $1444(44 \%)$ & $357(59 \%)$ \\
\hline Missing data, $N$ & 102 & 6 \\
\hline \multicolumn{3}{|l|}{ Leukopenia before admission } \\
\hline No & $3068(91 \%)$ & $561(92 \%)$ \\
\hline Yes & $320(9 \%)$ & $51(8 \%)$ \\
\hline 1-7 days & $212(6 \%)$ & $21(3 \%)$ \\
\hline$>7$ days & $108(3 \%)$ & $30(5 \%)$ \\
\hline \multicolumn{3}{|l|}{ Death in ICU } \\
\hline No & $2901(86 \%)$ & $528(86 \%)$ \\
\hline Yes & $487(14 \%)$ & $84(14 \%)$ \\
\hline \multicolumn{3}{|c|}{ Death at the hospital (including ICU) } \\
\hline No & $2836(84 \%)$ & $520(85 \%)$ \\
\hline Yes & $549(16 \%)$ & $92(15 \%)$ \\
\hline Missing data, $N$ & 3 & 0 \\
\hline
\end{tabular}

ICU intensive care unit, SAPS Simplified Acute Physiology Score, WHO World Health Organization, $q 25$ first quartile, $q 75$ third quartile

multivariate prognostic analyses are summarized in Table 3, and details are provided in the supplementary material (Tables S3-S6).

Among the 930 patients with MV, 45 were excluded from the prognostic analyses (10 had VAP) due to missing values among risk factors. VAPs were recorded for $143(16 \%)$ of the 
Fig. 1 Device utilization in the ICU at Gustave Roussy Hospital between 2000 and 2011
Number of stays with mechanical ventilator

(stays of $>=48 \mathrm{~h}$ )
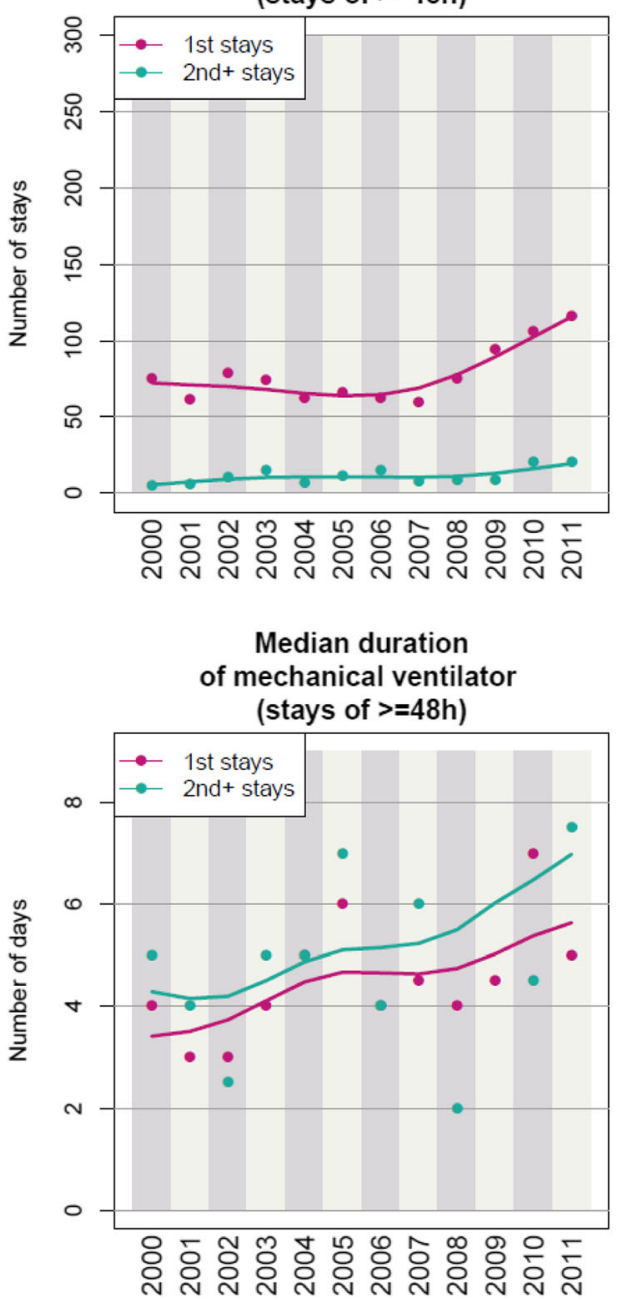

Number of stays with central venous catheters

(stays of $>=48 \mathrm{~h}$ )
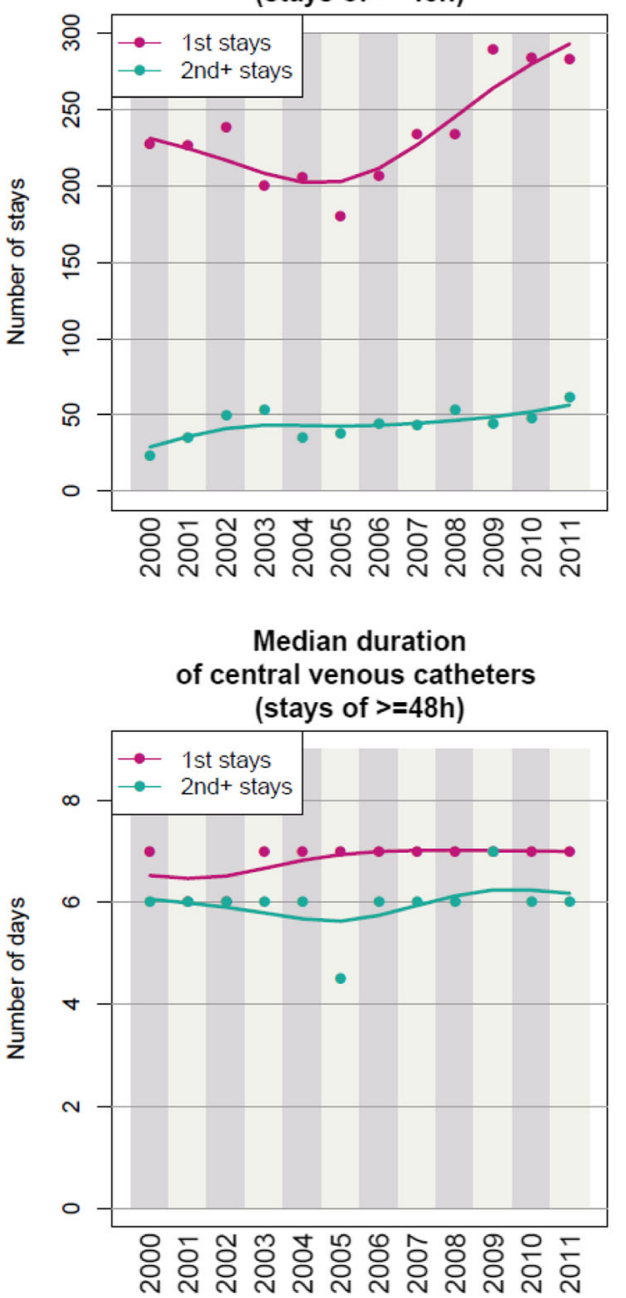

remaining 885 stays. The risk of developing VAP was constant over time. The cumulative risk of VAP after 25 days of MV was 58.8\% (95\% CI 49.1-66.6\%; Fig. 2). The duration of $\mathrm{MV}$, older age, scheduled surgery, and solid tumors were VAP-specific risk factors (Table S3).

Among the 3388 first stays, 134 were excluded from the prognostic analyses for the risk of BSI ( 2 had primary and 8 secondary BSIs) because of missing values among risk factors. Primary BSIs were recorded for 94 (3\%) of the remaining 3254 stays. The cumulative risk of primary BSI after a 25 -day ICU stay was $8.9 \%$ (95\% CI 6.2-11.5\%; Fig. 2). The length of stay, a high SAPS II score, scheduled surgery, ECOG-PS $>2$, absence of metastases, and recent leukopenia were significant risk factors (Table S4).

Secondary BSIs were recorded for $163 / 3254$ stays (5\%). The cumulative risk of secondary BSIs after a 25-day ICU stay was $15.1 \%$ (95\% CI 11.6-18.5\%; Fig. 2). The length of stay, surgery, and leukopenia were significant risk factors for secondary BSI (Table S5).
Among the 2806 patients with a CVC, 109 were excluded (1 had CR-BSI) because of missing risk factors. CR-BSIs were recorded for 72 (3\%) of the remaining 2697 stays. The cumulative risk of CR-BSIs after a 25-day CVC dwell time was $5.0 \%$ (95\% CI 3.2-6.8\%; Fig. 2). No significant risk factors were associated with the risk of CR-BSI, probably due to a lack of power (the low number of CR-BSIs; Table S6). Sensitivity analyses (Tables S7-S14) confirmed the robustness of these results.

\section{Mortality}

The occurrence of a VAP episode or BSI was not associated with a higher risk of ICU mortality in the univariate or multivariate analyses (Table S15). A significantly higher risk of death was observed for high SAPS II and ECOG-PS values, medical admission, and the presence of metastases. 
Table 2 Incidence of healthcare-associated infections in the intensive care unit

\begin{tabular}{|c|c|c|c|c|c|}
\hline & VAPs & pBSI & sBSI & CR-BSI & Total \\
\hline HAIs, $N$ & 198 & 103 & 213 & 77 & 591 \\
\hline Stays with at least one HAI, $N(\%)$ & $153(4.5)$ & $96(2.8)$ & $171(5)$ & $73(2.2)$ & $379(11.2)^{*}$ \\
\hline \multicolumn{6}{|c|}{ HAIs per 1000 ICU days, rate ( $95 \%$ conf. interval) } \\
\hline All patients & $6.3(5.4-7.2)$ & $3.3(2.7-3.9)$ & $6.7(5.8-7.6)$ & $2.4(1.9-3.0)$ & $18.7(17.2-20.2)$ \\
\hline Patients with leukopenia & $10.1(6.8-13.7)$ & $12.2(8.6-16.1)$ & $8.7(5.7-12.0)$ & $2.4(0.9-4.2)$ & $33.4(27.4-39.7)$ \\
\hline Patients without leukopenia & $5.8(4.9-6.7)$ & $2.2(1.7-2.8)$ & $6.5(5.6-7.5)$ & $2.4(1.8-3.0)$ & $16.9(15.4-18.4)$ \\
\hline \multicolumn{6}{|c|}{ HAIs per 1000 device days, rate ( $95 \%$ conf. interval) } \\
\hline All patients & $24.5(21.2-28.0)$ & - & - & $2.3(1.8-2.8)$ & - \\
\hline Patients with leukopenia & $24.1(16.3-32.6)$ & - & - & $1.8(0.7-3.1)$ & - \\
\hline Patients without leukopenia & $24.6(20.9-28.5)$ & - & - & $2.4(1.8-3.0)$ & - \\
\hline
\end{tabular}

The total duration of the ICU stays was 31,670 days; the total duration of the mechanical ventilator use was 8077 days; the total duration of central venous catheter use was 33,498 days

$H A I$ healthcare-associated infections, $I C U$ intensive care unit, $C I$ confidence interval, $V A P$ ventilator-associated pneumonia, $p B S I$ primary bloodstream infections, $s B S I$ secondary bloodstream infections, $C R$ - $B S I$ catheter-related bloodstream infections

*Not the row sum, but the number of ICU stays with at least one HAI of any type

$\$$ There were 3388 patients with a stay exceeding $48 \mathrm{~h}$

Fig. 2 Cumulative incidence of intensive care unit associated infection in the first 25 days of exposure. Black lines are one minus the Kaplan-Meier estimator, with their $95 \%$ confidence bands. Red lines are the exponential estimator, under the hypothesis of constant risk over time. In all these cases, the hypothesis of constant risk over time is compatible with $95 \%$ confidence bands of the nonparametric Kaplan-Meier curves
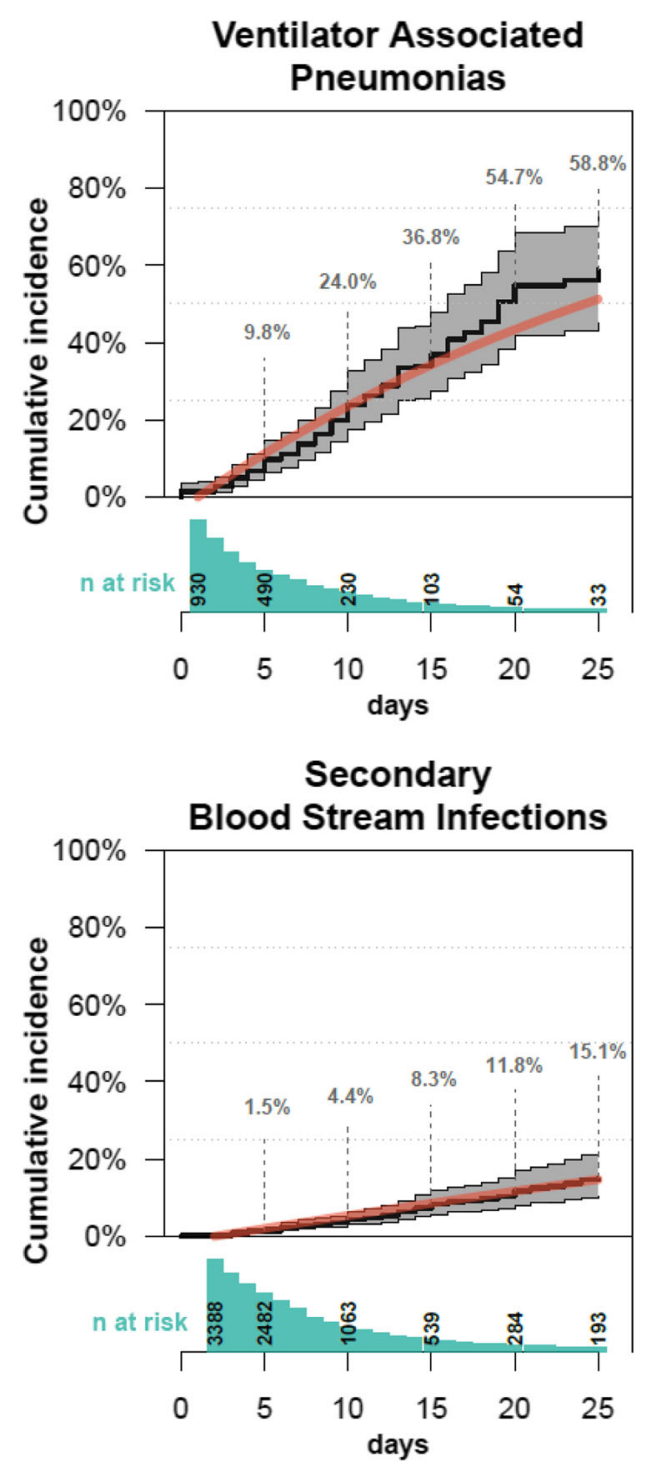
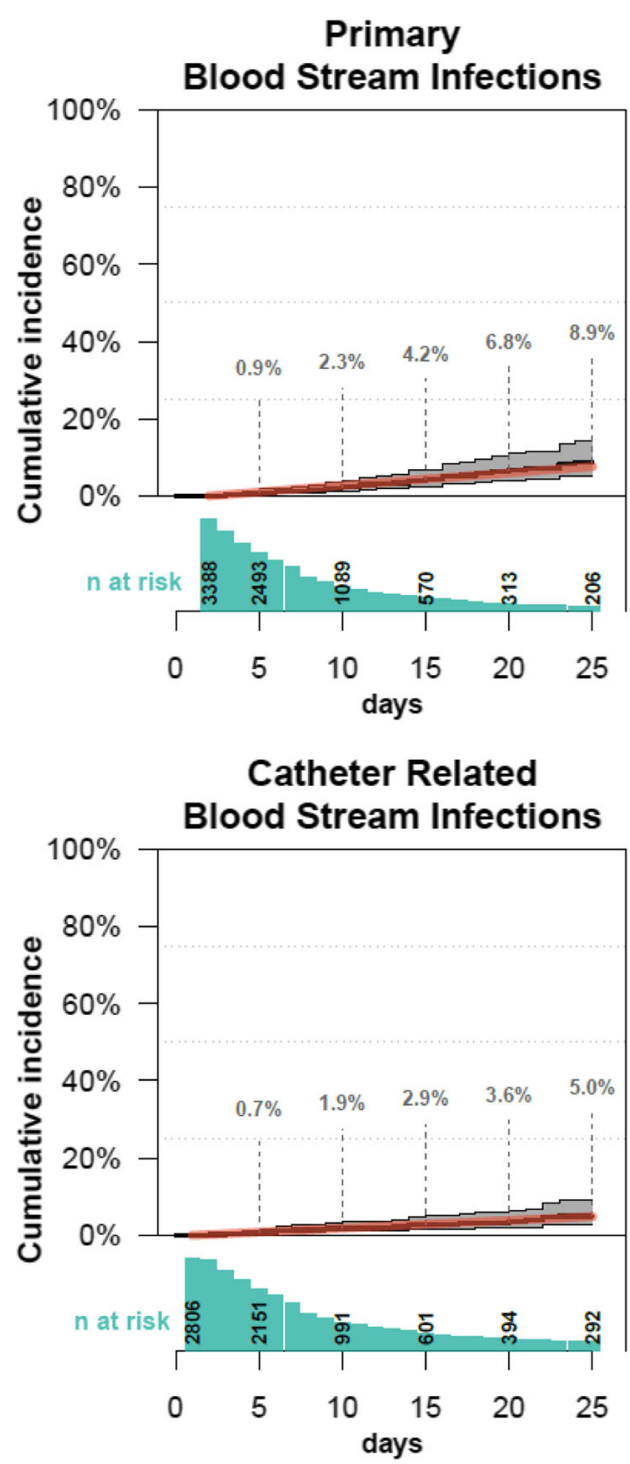


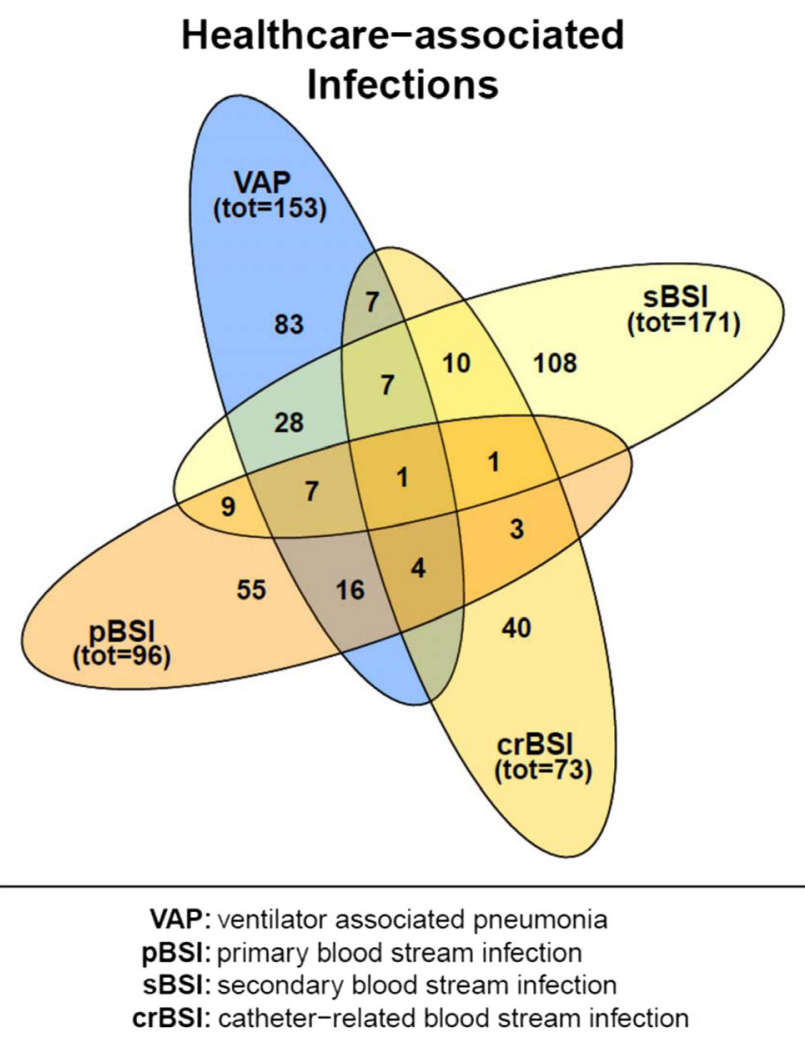

Fig. 3 Number of first stays in the intensive care unit (total =3388) with at least one of each type of infection. VAP ventilator-associated pneumonia, pBSI primary bloodstream infections, sBSI secondary bloodstream infections, CR-BSI catheter-related bloodstream infections

\section{Discussion}

We presented the results of a retrospective study on prospectively collected data, with drastic quality control measures which eliminated some inconsistencies. The stability of the admission categories, severity scores, and length of stay suggest that the case mix has not changed over time. To our knowledge, this study is the largest prospective series (3388; 12 years) on HAIs in cancer patients (see supplementary material). The study began after the introduction of alcoholbased hand gels. The main change in routine practice was the use of a sedation scale for mechanically ventilated patients since 2004.

Specific comments on device usage rates, slightly different from those reported previously, are given in the online supplementary material. Briefly, the rate of catheter use is higher (almost all of our patients have a long-term intravascular device) and the MV rate is lower [4] but has been increasing over time. As we focused on avoidable device-associated infections only, the overall incidence cannot be compared with other non-cancer populations.

We observed a higher VAP rate $(24.5 / 1000$ ventilator days) than in other studies $(12 / 1000$ at the end of the $90 \mathrm{~s}$ to $<6 / 1000$ during the past decade $[8,9]$ ), but lower than the VAP rate in the only study in cancer patients $(42 / 1000$ [4]). The incidence of VAP may have been underestimated given that microbiological samples were postponed when palliative care was decided, and some patients may have died with untreated pneumonia (or, similarly, with undiagnosed BSI...). Immunodeficiency due to malignancies and anticancer therapies can explain the high rate of VAP in cancer patients. However, neutropenia did not appear to be a risk factor for VAP, which is consistent with other studies $[10,11]$.

We observed a higher BSI rate (11.6/1000 ICU days) than that reported in a mixed population of French ICU patients $(4.5 / 1000)$ [12] and by the French National Surveillance Network [13] (3.53/1000). Our CR-BSI rate (2.3/1000 catheter days) is higher than those reported in the USA [14]. Mixing long-term and short-term CVCs in our study makes interpretation difficult: the REACAT study excluded long-term and pre-inserted CVCs. Interestingly, when CR-BSI secondary to long-term and short-term CVCs were examined in a post hoc analysis, a very similar infection rate was observed for catheters previously inserted when in the operating room (including totally implanted ports) and those inserted during the ICU stay. Unlike the steadily declining device-associated infection rates often reported $[8,15]$, our incidence of CRBSI increased between 2006 and 2009 and then decreased to below 2/1000 CVC days (Figure S3, right). We focused on CR-BSIs because they are more sensitive to preventive interventions than central line-associated BSIs and more relevant for comparisons between ICUs [16].

The distribution of germs is relatively close to that of general ICU populations, except for yeasts and anaerobic organisms. Candida species represented 5.5\% $(N=24$, including 11 cases of candidemia during leukopenia) of the isolated blood culture organisms, a rate that is similar to [8] or higher than [17] that of previous reports. Indeed, our population exhibited many recognized risk factors for candidemia [18]. Our high rate of anaerobic germs (13\%) is mainly due to the numerous heavy abdominal surgery cases admitted in our center.

Scheduled surgery and a solid tumor were VAPspecific risk factors, mainly due to hyperthermic intraoperative peritoneal chemotherapy (HIPEC) surgeries and esophagectomies, with regular complications requiring MV. Surgery (scheduled or not) and leukopenia were risk factors for secondary BSI, which could be due to HIPEC, often complicated by leukopenia and peritonitis. Nevertheless, after excluding 1051 scheduled surgery patients (Table S11), unscheduled surgery and leukopenia remained significant risk factors. Mucosal barrier injury (BSI), due to neutropenic enterocolitis, is not preventable and is classified as a secondary BSI in our study: in this case, leukopenia is not only a risk factor but also the 
Table 3 Prognostic factors for ventilator-associated pneumonia and bloodstream infections

\begin{tabular}{|c|c|c|c|c|c|c|c|c|c|}
\hline \multirow{3}{*}{$\begin{array}{l}\text { C-index } \\
\text { Prognostic factors }\end{array}$} & \multicolumn{3}{|l|}{ VAP } & \multicolumn{3}{|l|}{ pBSI } & \multicolumn{3}{|l|}{ sBSI } \\
\hline & \multicolumn{3}{|l|}{0.704} & \multicolumn{3}{|l|}{0.825} & \multicolumn{3}{|l|}{0.744} \\
\hline & $\operatorname{Pr}(\mathrm{VAP})$ & $95 \% \mathrm{CI}$ & $P$ & $\operatorname{Pr}(\mathrm{pBSI})$ & $95 \% \mathrm{CI}$ & $\mathrm{P}$ & $\operatorname{Pr}(\mathrm{sBSI})$ & $95 \% \mathrm{CI}$ & $P$ \\
\hline Exposure time & & & $<0.001$ & & & $<0.001$ & & & $<0.001$ \\
\hline 2 days & $10 \%$ & $8-12 \%$ & & $1 \%$ & $1-1 \%$ & & $2 \%$ & $1-2 \%$ & \\
\hline 8 days & $19 \%$ & $12-28 \%$ & & $2 \%$ & $1-4 \%$ & & $5 \%$ & $3-8 \%$ & \\
\hline Age (years) & & & 0.008 & & & & & & \\
\hline$\leq 50$ & $9 \%$ & $5-16 \%$ & & & & & & & \\
\hline $50-60$ & $13 \%$ & $7-22 \%$ & & & & & & & \\
\hline$>60$ & $18 \%$ & $11-26 \%$ & & & & & & & \\
\hline \multicolumn{10}{|l|}{ Sex } \\
\hline \multicolumn{10}{|l|}{ Male } \\
\hline Female & & & & & & & & & \\
\hline SAPS II & & & & & & 0.002 & & & \\
\hline$\leq 40$ & & & & $1 \%$ & $1-3 \%$ & & & & \\
\hline $40-50$ & & & & $2 \%$ & $1-5 \%$ & & & & \\
\hline$>50$ & & & & $4 \%$ & $1-8 \%$ & & & & \\
\hline Admission category & & & 0.006 & & & 0.02 & & & $<0.001$ \\
\hline Medical & $12 \%$ & $7-21 \%$ & & $1 \%$ & $0-2 \%$ & & $2 \%$ & $1-4 \%$ & \\
\hline Scheduled surgery & $22 \%$ & $12-35 \%$ & & $3 \%$ & $1-6 \%$ & & $6 \%$ & $3-9 \%$ & \\
\hline Unscheduled surgery & $11 \%$ & $5-21 \%$ & & $1 \%$ & $0-3 \%$ & & $6 \%$ & $3-11 \%$ & \\
\hline Type of neoplasia & & & 0.009 & & & & & & \\
\hline Solid tumor & $16 \%$ & $9-26 \%$ & & & & & & & \\
\hline Benign tumor & $2 \%$ & $0-11 \%$ & & & & & & & \\
\hline Hematological malignancy & $12 \%$ & $6-22 \%$ & & & & & & & \\
\hline Presence of metastases & & & & & & 0.01 & & & \\
\hline No & & & & $2 \%$ & $1-4 \%$ & & & & \\
\hline Yes & & & & $1 \%$ & $0-2 \%$ & & & & \\
\hline ECOG PS & & & & & & 0.02 & & & \\
\hline $0 / 1 / 2$ & & & & $1 \%$ & $1-3 \%$ & & & & \\
\hline $3 / 4$ & & & & $2 \%$ & $1-4 \%$ & & & & \\
\hline Leukopenia before admission & & & & & & $<0.001$ & & & 0.006 \\
\hline No & & & & $1 \%$ & $1-3 \%$ & & $3 \%$ & $2-5 \%$ & \\
\hline 1-7 days & & & & $7 \%$ & $3-15 \%$ & & $7 \%$ & $3-13 \%$ & \\
\hline$>7$ days & & & & $3 \%$ & $1-9 \%$ & & $8 \%$ & $4-17 \%$ & \\
\hline
\end{tabular}

Estimated probabilities (Pr) with their 95\% confidence interval (95\% CI) from univariate and multivariate analyses, adjusted for the ICU length of stay. For each category, the probability is computed for a mean profile of the other factors

$V A P$ ventilator associated pneumonia, $p B S I$ primary bloodstream infection, $S B S I$ secondary bloodstream infection, SAPS Simplified Acute Physiology Score, ECOG PS Eastern Cooperative Oncology Group performance status

cause of the BSI. The fact that ICU mortality was not influenced by the occurrence of VAPs or BSIs means that nosocomial infections mainly reflect the severity of the underlying disease or of the patient's condition. Nevertheless, we had no information on the adequacy of initial antimicrobial treatment, a key point in mortality attributable to VAP [19]. Finally, the design of our study does not allow a detailed analysis of the real prognostic burden of each HAI on mortality; aggregation of data into broad categories, such as VAPs and BSIs, lessens the actual impact of some types of HAI on the prognosis. Thus, the occurrence of a deep fungal infection in a neutropenic patient obviously has a greater impact on the prognosis than a catheter-related fungemia, for example. Identifying the mortality indeed attributable to each HAI (rather to underlying conditions) would require further analysis and studies.

\section{Conclusions}

The occurrence of device-associated infections is essentially due to severe medical conditions in patients and to the characteristics of the malignancy, but these infections do not influence the outcome of ICU cancer patients. Given the data were obtained in an oncology ICU in a specialized cancer center, extrapolation from these findings should be made very cautiously. However, these data may be useful for comparative studies with other oncology ICUs and for developing quality improvement activities. They could be also useful for comparison with HAI rates in the era of innovative treatments such as immunotherapy.

Acknowledgments We thank Monique Monhonval for data entry, Pascale Jan for technical support, and Lorna Saint Ange for editing. AS 
and FR had full access to all of the data in the study and take responsibility for the integrity of the data and the accuracy of the data analysis.

Author contributions $\mathrm{AS}$ and FB contributed to conception and design. AS, MW, and MM were involved in the data acquisition. FR and JPP planned and performed the statistical analyses. All the authors were involved in the interpretation of the results, read, and approved the final manuscript.

\section{Compliance with ethical standards}

Conflict of interest The authors declare that they have no conflict of interest.

\section{References}

1. Jarvis WR, Edwards JR, Culver DH, Hughes JM, Horan T, Emori TG, Banerjee S, Tolson J, Henderson T, Gaynes RP, Martone WJ, National Nosocomial Infections Surveillance System (1991) Nosocomial infection rates in adult and pediatric intensive care units in the United States. National Nosocomial Infections Surveillance System. Am J Med 91:185S-191S

2. Vincent JL, Bihari DJ, Suter PM et al (1995) The prevalence of nosocomial infection in intensive care units in Europe. Results of the European Prevalence of Infection in Intensive Care (EPIC) study. EPIC International Advisory Committee. JAMA 274:639644

3. Azoulay E, Soares M, Darmon M, Benoit D, Pastores S, Afessa B (2011) Intensive care of the cancer patient: recent achievements and remaining challenges. Ann Intensive Care 1:5

4. Velasco E, Thuler LC, Martins CA et al (1997) Nosocomial infections in an oncology intensive care unit. Am J Infect Control 25: 458-462

5. Hoheisel G, Lange S, Winkler J, Rodloff AC, Liebert UG, Niederwieser D, Schauer J, Engelmann L (2003) Nosocomial pneumonia in haematological malignancies in the medical intensive care unit. Pneumologie 57:73-77

6. Oken MM, Creech RH, Tormey DC, Horton J, Davis TE, McFadden ET, Carbone PP (1982) Toxicity and response criteria of the Eastern Cooperative Oncology Group. Am J Clin Oncol 5: $649-655$

7. Le Gall JR, Lemeshow S, Saulnier F (1993) A new simplified acute physiology score (SAPS II) based on a European/North American multicenter study. JAMA 270:2957-2963

8. NNIS System (1999) National Nosocomial Infections Surveillance (NNIS) System Report, data summary from January 1990-May 1999, issued June 1999. A report from the NNIS System. Am J Infect Control 27:520-532

9. Zack JE, Garrison T, Trovillion E, Clinkscale D, Coopersmith CM, Fraser VJ, Kollef MH (2002) Effect of an education program aimed at reducing the occurrence of ventilator-associated pneumonia. Crit Care Med 30:2407-2412

10. Groeger JS, Bach PB (2003) Consider saying yes. Crit Care Med 31:320-321

11. Mokart D, Darmon M, Resche-Rigon M, Lemiale V, Pène F, Mayaux J, Rabbat A, Kouatchet A, Vincent F, Nyunga M, Bruneel F, Lebert C, Perez P, Renault A, Hamidfar R, Jourdain M, Meert AP, Benoit D, Chevret S, Azoulay E (2015) Prognosis of neutropenic patients admitted to the intensive care unit. Intensive Care Med 41:296-303

12. Renaud B, Brun-Buisson C (2001) Outcomes of primary and catheter-related bacteremia. A cohort and case-control study in critically ill patients. Am J Respir Crit Care Med 163:1584-1590

13. Surveillance des infections nosocomiales en réanimation adulte (2016) Réseau REA-Raisin, France - Résultats 2014. SaintMaurice : Institut de veille sanitaire. 48 p. http://www.invs.sante. fr/Publications-et-outils/Rapports-et-syntheses/ Maladiesinfectieuses/2016/Surveillance-des-infectionsnosocomiales-en-reanimation-adulte

14. Pronovost PJ, Goeschel CA, Colantuoni E, Watson S, Lubomski LH, Berenholtz SM, Thompson DA, Sinopoli DJ, Cosgrove S, Sexton JB, Marsteller JA, Hyzy RC, Welsh R, Posa P, Schumacher K, Needham D (2010) Sustaining reductions in catheter related bloodstream infections in Michigan intensive care units: observational study. BMJ 340:c309

15. Dudeck MA, Horan TC, Peterson KD, Allen-Bridson K, Morrell G, Pollock DA, Edwards JR (2011) National Healthcare Safety Network (NHSN) report, data summary for 2010, deviceassociated module. Am J Infect Control 39:798-816

16. Sihler KC, Chenoweth C, Zalewski C, Wahl W, Hyzy R, Napolitano LM (2010) Catheter-related vs. catheter-associated blood stream infections in the intensive care unit: incidence, microbiology, and implications. Surg Infect 11:529-534

17. Brun-Buisson C, Doyon F, Carlet J (1996) Bacteremia and severe sepsis in adults: a multicenter prospective survey in ICUs and wards of 24 hospitals. French Bacteremia-Sepsis Study Group. Am J Respir Crit Care Med 154:617-624

18. Talarmin JP, Boutoille $D$, Tattevin $P$, Dargère $S$, Weinbreck $P$, Ansart S, Chennebault JM, Hutin P, Léautez-Nainville S, GayAndrieu F, Raffi F (2009) Epidemiology of candidemia: a oneyear prospective observational study in the west of France. Med Mal Infect 39:877-885

19. Agrafiotis M, Siempos II, Ntaidou TK, Falagas ME (2011) Attributable mortality of ventilator-associated pneumonia: a metaanalysis. Int J Tuberc Lung Dis 15:1154-1163

Publisher's note Springer Nature remains neutral with regard to jurisdictional claims in published maps and institutional affiliations. 